Miguel A. Márquez

Facultad de Medicina Veterinaria y Zootecnia Universidad Nacional Autónoma de México Sociedad Mexicana de Historia de la Medicina Veterinaria y Zootecnia

*Autor para correspondencia: Correo electrónico: miguel.marquez@unam.mx

\title{
Pascal Eugène Bergeyre Lagrange La Aventura Mexicana del Primer Médico Veterinario Civil y Militar de México (1829-1880)
}

\section{Al primero, la historia nunca le olvida}

El científico francés Eugène Bergeyre tiene el honor de haber sido el primer veterinario que arribó y ejerció la medicina veterinaria como disciplina científica en México. Pascal Eugène Bergeyre Lagrange, nace un 23 de febrero de 1829 en la ciudad de Bayona, capital del País Vasco francés, ubicada en el Suroeste de Francia, ya muy cerca de la frontera con España en lo que actualmente es el Departamento de los Bajos Pirineos Atlánticos. Sus progenitores fueron Antoine Bergèyre y Jeanne-Etienette Lagrange. Fue el sexto vástago de ocho hermanos (cuatro varones y tres mujeres). ${ }^{1}$

Procedía de una larga saga de maréchaux-ferrants (mariscales-herradores), línea que se inicia en Arthez-de-Béarn (Pirineos Atlánticos) hacia 1680 con Daniel Bergeyre, alias Bascou, y que se continua con Bernard Pascal Bergeyre, su abuelo paterno, quien laboró como marechal-ferrant, y Antoine, su padre, quien ejerció ya como artiste vétérinaire en la campiña bayonesa. Su madre, Jeanne Lagrange, era a su vez, hija de un comerciante de caballos de Bayona. ${ }^{2}$

Además, su hermano mayor, Jean Baptiste Prosper Bergeyre, se desempeñó también como un distinguido veterinario, al heredar la clínica y clientela de su padre en Bayona. De esta manera, tatarabuelo, abuelo, padre y hermano, así como las verdes praderas que cubren la confluencia de los ríos Nive y Adur, debieron marcar indeleble y profundamente la mente infantil y juvenil d'Eugène, experiencias que lo condujeron a cursar estudios de medicina animal en l'École Nationale Vétérinaire de Tolosa, al Sur de Francia.

\section{Estudios de Medicina Veterinaria}

El 16 de octubre de 1846 ingresa a la Escuela Veterinaria de Tolosa, en donde al paso de cuatro años de estudios obtiene su Diploma de Veterinario (Figura 1), después de hacer la defensa de sus conocimientos y habilidades ante un jurado integrado por cuatro profesores el 21 de agosto de 1850, quienes declaran que:

\section{Cite this as:}

Márquez MA. Pascal Eugène Bergeyre Lagrange. La Aventura Mexicana del Primer Médico Veterinario Civil y Militar de México (1829-1880). Veterinaria México OA. 2020;7(4). doi: 10.22201/fmvz.24486760e.2020.4.956. 
El jurado, después de haber interrogado al señor Bergeyre (Pascal Eugène), sobre todas las diferentes ramas de la medicina veterinaria de los animales domésticos, de haberle hecho practicar diversas operaciones tanto quirúrgicas, como farmacéuticas, ha reconocido que él se encuentra en estado de cumplir las funciones de Veterinario.

Razón por la cual se la ha entregado la presente constancia, en virtud de que el señor Bergeyre (Pascal Eugène) esta autorizado a ejercer la Medicina Veterinaria.

Dado en la Escuela Veterinaria de Toulouse, el 21 de agosto de 1850". ${ }^{3}$

\section{Recién egresado}

Disponemos de poca información sobre la actividad profesional del joven Bergeyre, como veterinario recién graduado entre 1850 a 1853. Sólo sabemos que fue miembro de la Societé Agricole de la Charente Maritime (Sociedad Agrícola de la Carente Marítima), en donde debió laborar, suponemos, como veterinario de campo, antes de emprender el viaje hacia América.

Como hemos documentado previamente, Pascal Eugène obtuvo su licencia que le permitió ejercer su profesión como veterinario en Francia en 1850, a la edad de 21 años, cuando Carlos Luis Napoleón Bonaparte gobernaba como el primer y único presidente de la Segunda República Francesa (1848-1851). El futuro Napoleón III, sobrino del gran Napoleón I, se aprestaba a dar el golpe de Estado que le convertiría, en 1852, en Emperador de los Franceses, constituyéndose así el II Imperio de Francia que duraría hasta 1870.

Por otro lado, en la República Mexicana a mediados del siglo XIX, el presidente en turno era don José Joaquín Antonio Florencio de Herrera y Ricardos (1792-1854), político y destacado militar que ocupara en tres ocasiones la presidencia. El país emergía con enormes dificultades de la traumática e injusta invasión estadounidense que, a través del Tratado de Guadalupe Hidalgo, le había despojado y amputado más de dos millones de kilómetros cuadrados de su territorio Norte. Las luchas entre liberales y conservadores volvían a resurgir y a enconarse, pero México era un país en el cual todo estaba por hacerse y que ofrecía grandes oportunidades para trabajar, para iniciar cualquier tipo de negocios y amasar grandes fortunas.

Dentro de este marco de eventos, debemos encontrar o suponer las razones por las cuales Eugène Bergeyre tomó la decisión de dejar todo, familia y país, con el objeto hacer una nueva vida en un país desconocido.

\section{¿Por que razones Eugène Bergeyre emigró a México?}

¿Qué motivos lo hicieron emigrar? ¿Qué ilusiones o carencias lo impulsaron a embarcarse a un país tan diferente y ajeno a él? ¿Qué buscaba? ¿Fue el espíritu de aventura? ¿La ausencia de un futuro prometedor en su país natal? ¿El deseo o la imagen de un país y una sociedad donde podría hacer fortuna rápidamente? 

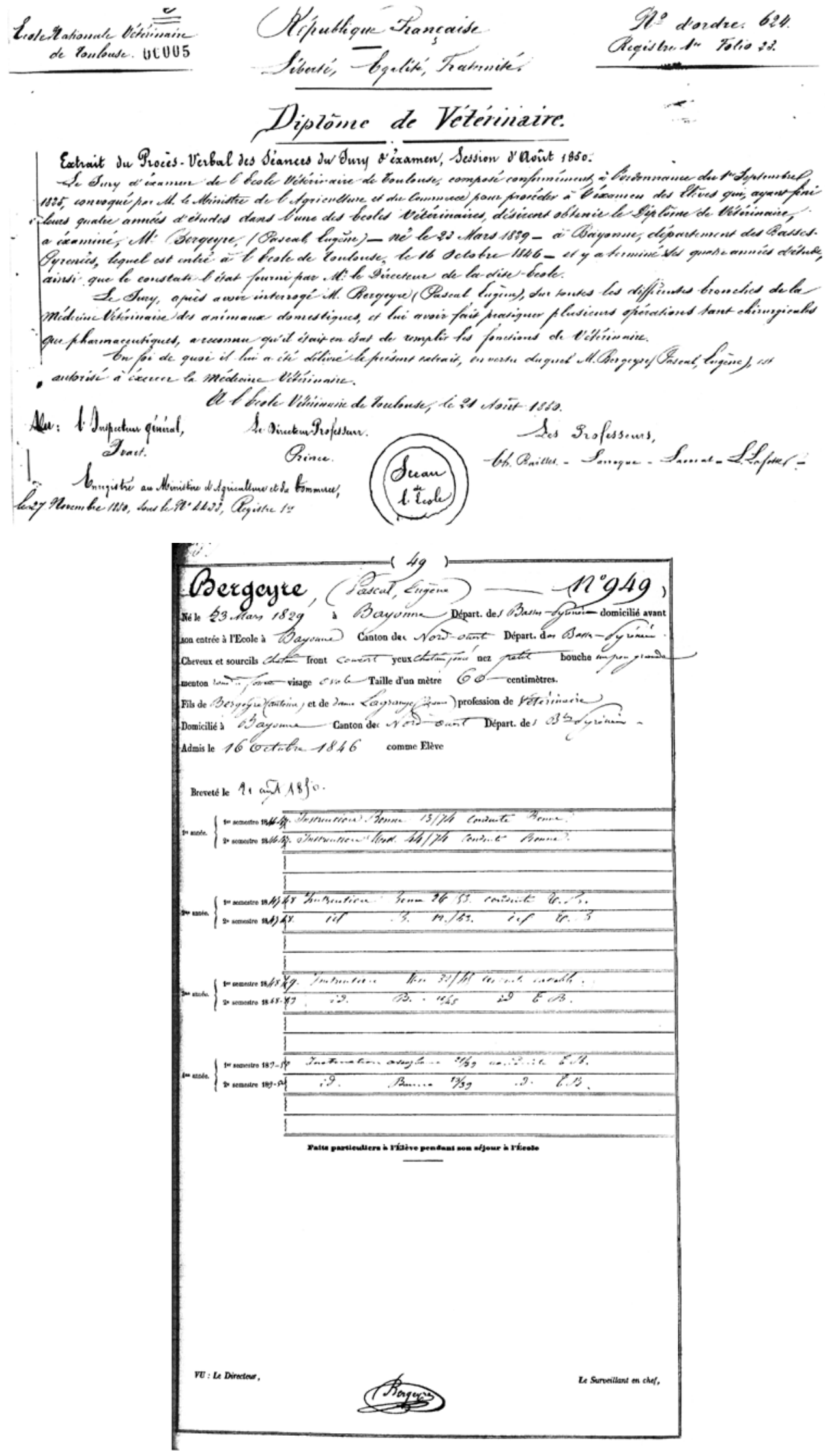

Figura 1. Expediente escolar del alumno Pascal Eugène Bergeyre Lagrange. Escuela Nacional de Veterinaria de Tolosa, Francia, 1850. (Documento proporcionado amablemente por el Sr. Daniel Wiart; 2009.) 
Una razón poderosa y comprensible para establecerse en México fue que Víctor, su hermano menor, había emigrado a la capital de este país en 1848, donde se ganaba la vida como "guarnicionero" o talabartero, manufacturando e importando arneses para caballos y sillas de montar. Esta actividad sería continuada años después por su sobrino Pascal Víctor Bergeyre (hijo de Eugène) a finales del siglo XIX y principios del XX.

La siguiente huella documental que encontramos de nuestro personaje es una larga carta dirigida a sus padres, fechada en ciudad de México, el $1^{\circ}$ de julio de 1853, escrita apenas arribado a ciudad de México, a la edad de 24 años y dirigida a sus padres. ${ }^{4}$

Además de ser una misiva de noticias de carácter familiar, parece ser un fascinante diario de viaje, pues en ella relata las vicisitudes, alegrías y sufrimientos que vivió durante larga y penosa travesía marítima en el navío velero Panamá, los avatares y los peligros del duro ascenso en una diligencia tirada por ocho caballos, hasta la capital mexicana, desde el puerto de Veracruz, cuya población en el momento de su arribo estaba siendo devastada por una epidemia de Cholera morbus ${ }^{\text {a }}$.

Esta carta es por demás reveladora, ya que hacia la parte final de ella, el texto menciona: "le mois prochain [...] vous saurez amplement quelle sera ma position" (el mes próximo [...] ustedes sabrán ampliamente cuál será mi posición).

Wiart y Cabantous, expertos genealogistas franceses, que han estudiado a la familia Bergeyre y quienes publicaron la mencionada carta en la revista del Cercle Génealogique du Languedoc, escriben en su artículo a pie de página que: "Eugene démarre son activité a Mexico ayant pour objétif: amasser un pecule en cinq ou six ans, rembouser les dettes contractées avant son départ et revenir une fois fortune faite". ["Eugene inicia su actividad en México con el objetivo de reunir un caudal en cinco o seis años, pagar las deudas contraídas antes de su partida y retornar una vez hecha una fortuna"]. ${ }^{5}$

Todo esto prueba que Bergeyre llegó a México por su propia iniciativa y sin tener una posición previa de ofrecimiento de empleo. Todo indica, por lo tanto, que nuestro biografiado vino a establecerse para ejercer su profesión de veterinario en una ciudad y en un país donde no existían servicios de esta índole. Tan sólo un mes después de su arribo a la capital mexicana, Bergeyre se hace anunciar en tEl Siglo Diez y Nueve, uno de los principales diarios del siglo XIX en México.

El lunes $1^{\circ}$ de agosto de 1853, aparece en el mencionado periódico, el siguiente anuncio (Figura 2):

a El propio Bergyere acota en su carta que: On a surnommé Vera-Cruz: "le tombeau des Europeans", et c'est avec trop de raison. Si j'y étais reste quelques jours, la crainte de ne pas voir mon bon frère Victor aurait finit par me faire craindre que j'étais atteint de quelqu'une de ces maladies... (Se ha llamado a Veracruz: "la tumba de los Europeos" y con mucha razón. Si yo me hubiera quedado algunos días, el miedo de no ver a mi buen hermano Víctor, habría terminado por hacerme temer que yo estaba enfermo de alguna de esas enfermedades...) 


\section{AL PÚBLICO}

EUGENIO BERGEYRE, médico veterinario de la Facultad de Medicina Veterinaria de Tolosa (Francia), miembro de la Sociedad de Agricultura de la Carente, tiene el honor de anunciar à las personas que gusten otorgarle con su confianza, que acaba de abrir un establecimiento en la calle de Victoria num. 14, en donde se dedicará al tratamiento y curación de todas las enfermedades de que adolecen los animales domésticos, como son la raza caballar, canina y borina, etc., etc.

En el mismo establecimiento se encuentra un banco de herrador y se reciben caballos a pensión. ${ }^{6}$

$307-10-1$

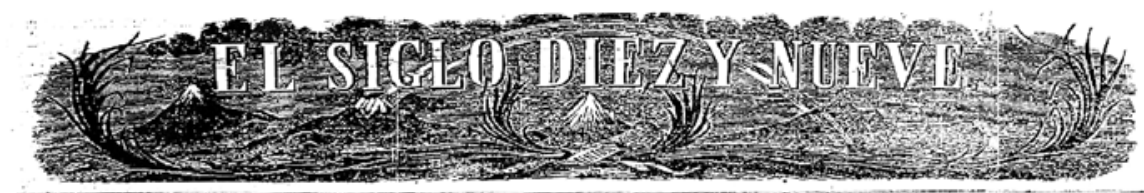

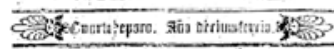

MEAace, LE XIS 10 DE AG0STO DE 1853.

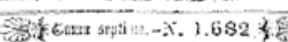

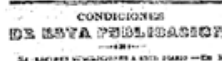

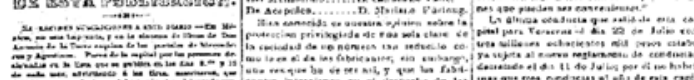
$+2=0$

$=$

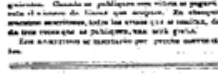

\section{EMTTOREAR.}

REYISTA MFERCANTI

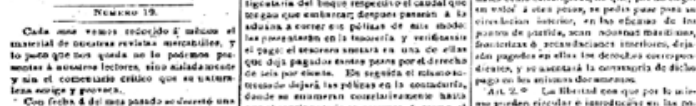

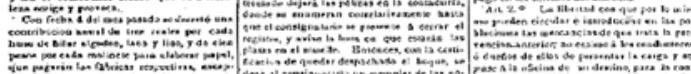

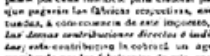

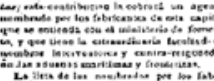

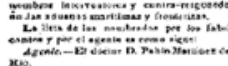

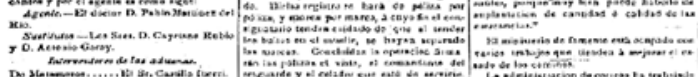

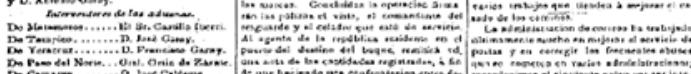

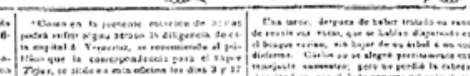

10.

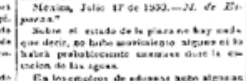

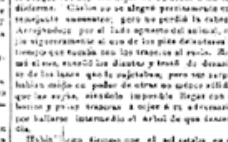
-

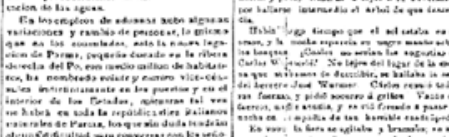

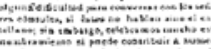
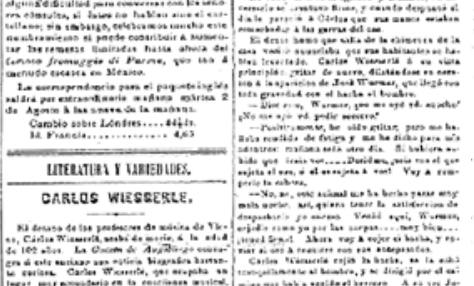

踏

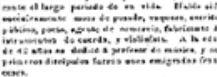
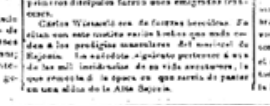
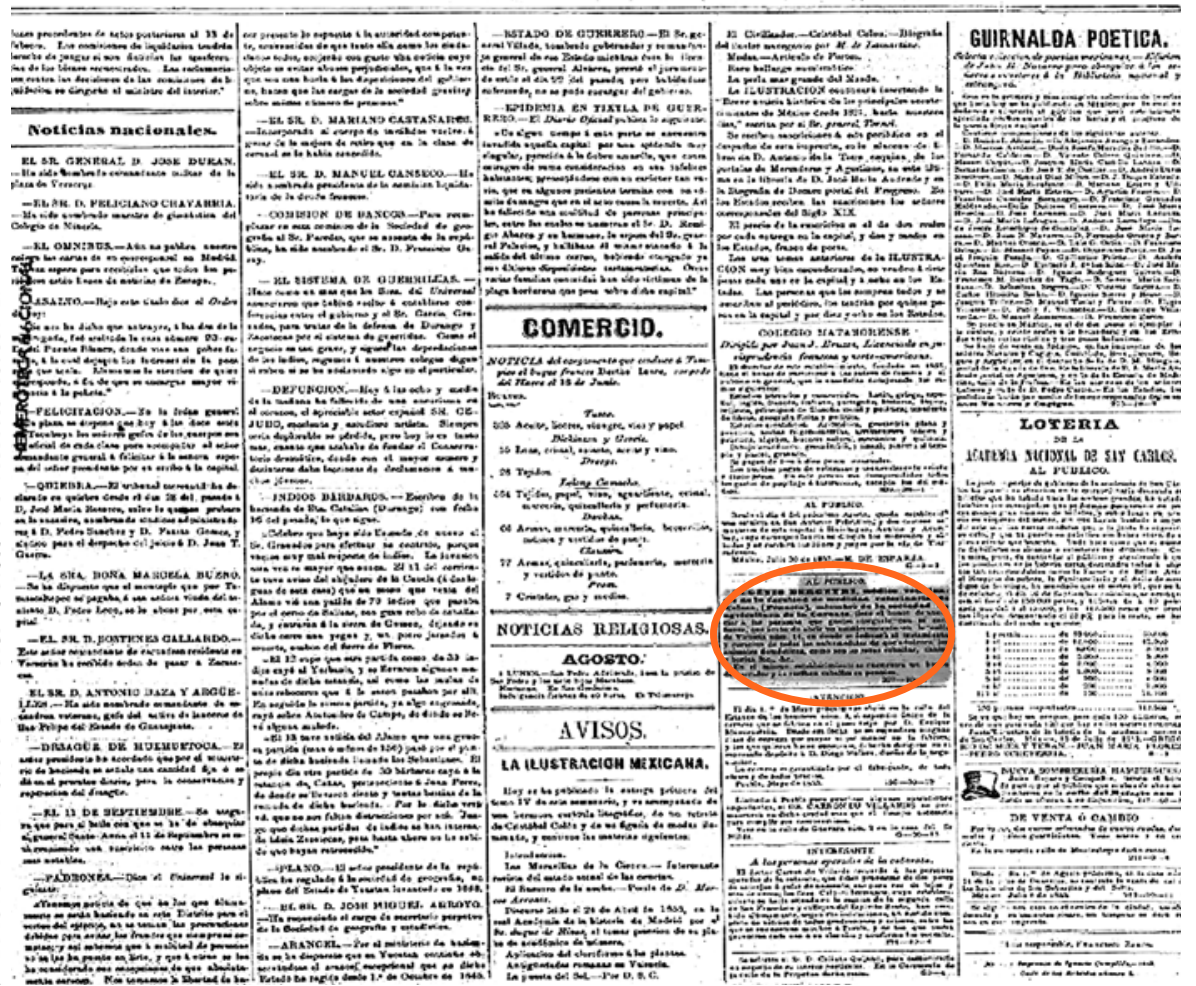

Figura 2. Página del Diario EL SIGLO DIEZ Y NUEVE en donde aparece el anuncio del Dr. Eugenio Bergeyre ofreciendo sus servicios como profesional veterinario, además de los servicios de herrado y pensión para caballos. 
La misma publicidad apareció en nueve ocasiones entre el $1^{\circ}$ de agosto y el 9 de septiembre del mismo año. Este anuncio tiene una gran relevancia, pues es probablemente la primera publicidad en la cual un profesional de la medicina veterinaria ofrezca sus servicios privados a los habitantes de la ciudad de México y seguramente se trate además de la primera clínica privada para la atención y curación de enfermedades grandes animales y perros. ${ }^{7}$

Eugenio trabaja seriamente y pronto tiene en su cuadra más 18 caballos para curar y un compatriota galo le permite obtener un contrato con la compañía de diligencias.

\section{La caída de un mito}

Según la información antes descrita, es inevitable echar por tierra el mito que todos los veterinarios mexicanos manejábamos con orgullo desde hacía tantos años: el de que, el Generalísimo Antonio López de Santa Anna, en ese entonces presidente de la República Mexicana, había solicitado al gobierno francés a un veterinario militar experto y que el ejército francés habría enviado a Eugène Bergeyre, comisionado a México para hacerse cargo del cuidado de los caballos de la cuadra presidencial y, de manera particular, de los gallos de pelea del presidente.

La esposa del primer embajador plenipotenciario de España en México después de la guerra de independencia, y del reconocimiento de España a la nación mexicana como país independiente, madame Calderón de la Barca, relata de exquisita manera en 1838, la afición del general Santa Anna por los gallos de pelea, en ocasión de su visita a la Hacienda Manga de Clavo, en Veracruz, durante su viaje de ascenso en diligencia a la ciudad de México:

En attendant, se anunció el almuerzo. La Señora de Santa Anna me introdujo al comedor. Colocaron a Calderón a la cabecera y a mí a su derecha; Santa Anna enfrente de Calderón, y la Señora a mi derecha. El almuerzo fue espléndido, y consistió en una variedad de platos españoles, carne y legumbres, pescado, aves, frutas, y dulces, café, y vinos, etcétera, todo servido en vajilla francesa en blanco y oro. Después del almuerzo [...] vimos las dependencias y las oficinas, y también el caballo de batalla predilecto del general, un viejo corcel blanco, quizá un filósofo más sincero que su amo; varios gallos de pelea, criados con especial cuidado, ya que las peleas de gallo son unas de las diversiones favoritas de Santa Anna... 8

\section{El presidente Santa Anna, la fundación del primer centro de educación veterinaria en América y el Dr. Bergeyre}

El presidente Santa Anna fundó el Colegio Nacional de Agricultura y Veterinaria, por medio del decreto presidencial número 4001, fechado el 17 de agosto del año de 1853 en el Palacio de Gobierno de la villa de Tacubaya, Pasarían; sin embargo, algunos años para que dicha institución, sus alumnos, el profesorado y los cursos tomaran cuerpo físicamente y fueran operativos. Los cursos agricultura inician en 
febrero de 1854 y los de veterinaria lo hacen hasta enero de 1856, en el vetusto edificio del antiguo convento dominico de San Jacinto, ubicado en el barrio de Tacuba, al poniente de la ciudad de México.

Ya en un listado de profesores y empleados del año 1857 aparece el Dr. Eugenio Bergeyre como propietario de la Cátedra Mariscalía y Exterior. ${ }^{9}$

Asimismo, Manuel Ramírez Valenzuela, eminente bacteriólogo, higienista e historiador de la veterinaria, cita a Bergeyre de la manera siguiente:

En lo que respecta a la enseñanza de la Medicina Veterinaria, el Dr. Río de la Lozab, no tuvo problemas, pues las cátedras, estaban a cargo del competente veterinario militar francés, el Dr. Eugenio Bergeyre, quien radicaba en México desde 1853. El Dr. Bergeyre tuvo a su cargo las cátedras de Delineación, Anatomía, Farmacología, Mariscalía y Exterior, Patología, Cirugía Veterinaria, siendo por lo tanto, el primer médico veterinario que tuvo la escuela como profesor y que formó científica y moralmente a las primeras generaciones de médicos veterinarios que constituirían los cimientos de la profesión en México. ${ }^{10}$

Finalmente, Flores Troncoso, el médico y erudito historiador de la medicina de nuestro país de fines del siglo XIX, cita en su monumental y riquísima tesis que:

En 1851, durante la memorable administración del gobierno del General Arista, se volvió a pensar en la fundación de una Escuela de Agricultura aunque sin lograrlo, hasta que, por fin, habiendo llegado a México, en el año de 1853 un veterinario francés de la Escuela de Tolosa, el Sr. Eugenio Bergeyre, y habiendo servido en el ejército mexicano, y tenido especialmente a su cargo las caballerizas del presidente, General Santa Anna, se vio la conveniencia de la fundación de esta Escuela. ${ }^{11}$,

\section{De la relación profesional y personal entre el Generalísimo Antonio López de Santa Anna y el Dr. Eugenio Bergeyre}

Al abrir su consultorio, su pensión para caballos y el banco de herrado en la calle de Victoria No. 14, Eugenio Bergéyre debió de haberse hecho rápidamente de un prestigio entre la alta sociedad capitalina, el cual debió haber llegado a los oídos del presidente.

En este contexto, es probable que Bergèyre fuera contactado e invitado por Santa Anna para hacerse cargo de los caballos de la cuadra presidencial.

b El Dr. Leopoldo Río de la Loza, químico, médico y distinguido científico mexicano, fue el primer director de la Escuela Nacional de Agricultura y Veterinaria.

c Hemos buscado en diversas fuentes, pero principalmente en el rico expediente que existe del Dr. Bergèyre en el Archivo Histórico de la Secretaría de la Defensa Nacional en ciudad de México, y no fuimos capaces de encontrar un documento que pruebe que nuestro personaje biografiado haya ingresado al ejército mexicano, sin embargo, la mención que hace Flores Troncoso en su obra, nos basta para documentar y probar el alta y la presencia de Bergeyre en el ejército de México. 
Asimismo, no hemos sido capaces de encontrar un documento que pruebe que Eugenio Bergeyre haya causado alta en el ejército mexicano en tiempos del General Santa Anna o en los de sus sucesores. Sin embargo, no se duda del hecho que hubiera atendido la caballeriza presidencial, pues era el profesional adecuado para hacerlo.

Un señalamiento más que resalta la enorme importancia con la que se revistió el Dr. Bergeyre, como profesor fundador y miembro de la primera orla de maestros y del papel protagónico que desempeñó en la fundación de la primera Escuela de Agricultura y Veterinaria, es el mencionado por los colegas veterinarios e historiadores Cervantes, Román, López y Uribe:

Debido a la concepción médica que se tenía de la profesión, encaminada principalmente a la cura del caballo, se dice que en 1853 Santa Anna recibió la idea de fundar la primera escuela de veterinaria en México, del veterinario Eugenio Bergeyre; todo parece indicar que este médico francés es quien hace notar al presidente de la conveniencia de fundar una escuela de veterinaria en el país, pues hasta entonces no existía ninguna escuela en su tipo y la medicina veterinaria no figuraba como una disciplina científica. ${ }^{12,13}$

En el expediente de Bergèyre, que existe en el Archivo Histórico de la Secretaría de la Defensa Nacional, se halla un folio con un listado escrito con elegante letra de estilo Palmer, encabezada de la manera siguiente: "Documentos acompañando al Diploma de Veterinario" (Figura 3).

En dicho listado se enumeran cuatro documentos:

1. "Nombramiento de Profesor de Veterinaria, expedido por el General Don Antonio López de Santa Anna". No se menciona fecha. Este documento se encuentra extraviado.

2. "Un nombramiento de Veterinario Militar francés". Se trata del valioso documento por medio del cual, el Emperador Maximiliano nombra a Bergyere, Veterinario Principal del ejército de ocupación francés.

3. "Un nombramiento de Socio de la Comisión Científica de México". Extraviado.

4. "Un nombramiento de Inspector de Carnes". Extraviado.

\section{Los vientos del cambio soplan de Europa}

Entre los años de 1853 y 1864, nuestro biografiado debió dividir su vida profesional como profesor de la Escuela Nacional de Agricultura y Veterinaria de San Jacinto, su posición en el ejército y su práctica privada en su clínica veterinaria de la calle de Victoria. ${ }^{14}$ 


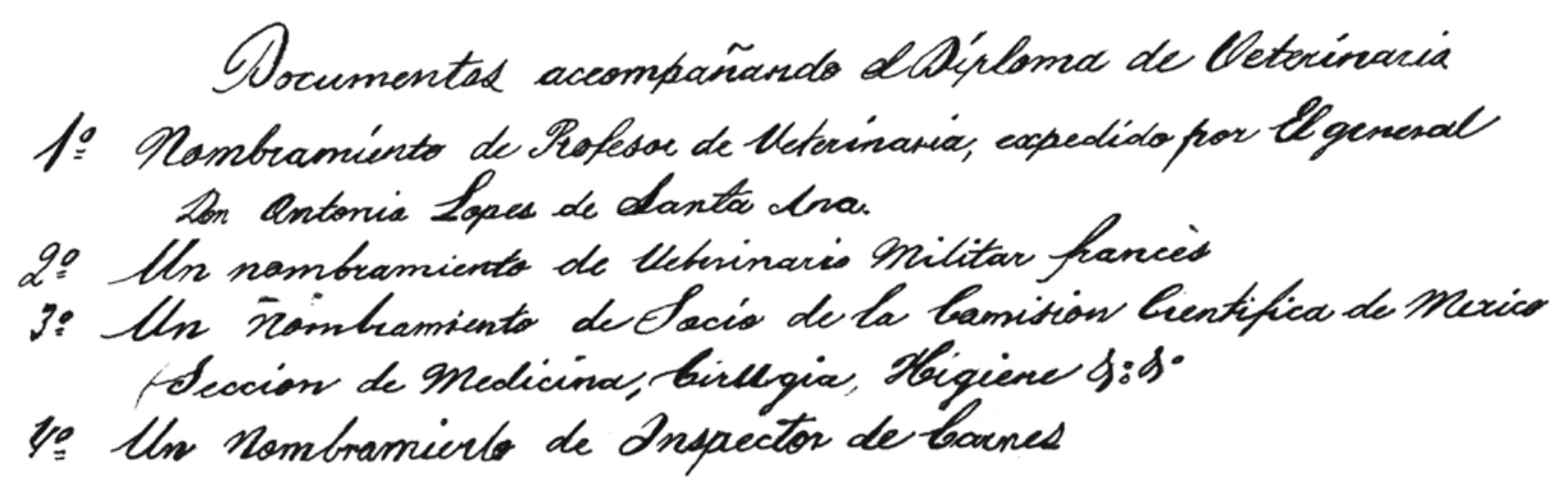

Figura 3. Documentos acompañando al Diploma de Veterinario.

La Revolución de Ayutla, comandada por el caudillo sureño Juan Álvarez, había expulsado del país a Santa Anna en 1855, quien partió hacia el exilio colombiano, por lo que Eugenio pierde a su amigo y mecenas. Los gobiernos liberales de Ignacio Comonfort y Benito Juárez fueron vencidos por sus acérrimos enemigos, los conservadores, a lo largo de interminables guerras intestinas. Las armas y los gobiernos de Félix María Zuloaga, Miguel Miramón y Juan Nepomuceno Almonte, se imponen temporalmente sobre ellos, entre 1858 y 1864. Los mexicanos y la población extranjera avecindada, sobre todo, en ciudad de México, deben aprender a vivir y adaptarse a cada voltereta de la política nacional y a las penurias y vicisitudes de la endémica guerra civil. Así lo hacen, Eugenio y su hermano Víctor. Once años han transcurrido desde su arribo.

\section{El Segundo Imperio Maximiliano de Habsburgo, Emperador de México}

La derrota del reputado ejercito francés comandado por el conde de Lorencez, ante los muros de los fuertes de Loreto y Guadalupe en Puebla, en mayo del 1862, la caída de la capital mexicana en manos del ejército expedicionario francés del Mariscal Forey en junio de 1863 y la regencia imperial de Juan Nepomuceno Almonte, son preludio a la recepción que ofrece la ciudad de México, la mañana del 12 de junio de 1864, a sus altezas reales, el emperador Fernando Maximiliano José de Habsburgo-Lothringen y a la emperatriz Carlota Amalia de Bélgica. La condesa austriaca Paula von Kolonitz, dama del séquito de Carlota, menciona que duró tres días y la calificó como apoteótica: 
La comitiva se detuvo ante la Catedral, que ocupa la segunda parte de la gran plaza, a la derecha del Palacio. Aquí se cantó un Te Deum y después todo el cortejo recorrió a pie un camino cubierto de alfombras y bajo una tienda, hacia la residencia. Banderas y miles y miles de guirnaldas de bellísimas flores adornaban la entrada, en la que se veían los retratos de sus majestades, bastante mal pintados. Una enorme multitud cubría la grandísima plaza, pero el orden y la quietud reinaban en todos lados; los mexicanos y los indios no son impacientes, ni ruidosos. Mucha alegría y mucho interés demostró el pueblo y el saludo de México fue cordialísimo... ${ }^{15}$

De esta manera aparentemente promisoria comienza el Segundo Imperio. Los mil días que gobernará el joven y bien intencionado archiduque liberal de la Casa de los Austrias constituyeron un mandato complejísimo, marcado por la tragedia y destinado al fracaso y durante el cual, Eugenio Bergéyre desempeñó un papel protagónico como militar y científico.

Como súbdito del emperador Napoleón III, y como profesional experimentado en la medicina animal y diestro en el manejo de los caballos, era inevitable que Eugenio se incorporara al ejército imperial mexicano.

A este respecto, hemos podido estudiar dos documentos, que sostuvieron entre sus manos, tanto el Emperador Maximiliano, como el Dr. Bergeyre.

El primero es una carta escrita en español que dirige Dr. Eugenio Bergeyre, Ayudante Veterinario de Segunda Clase del Ejército Francés, al Inspector General del Ministerio de Guerra del Segundo Imperio, el 23 de agosto de 1866 y en la cual solicita el cargo de Veterinario Principal. La respuesta dada cinco días más tarde, se halla escrita en la parte inferior del mismo folio, con fecha del 28 de agosto del mismo año, en la que se le otorga, en efecto, el cargo de Veterinario del Ejército Francés (Cuerpo Expedicionario Francés de ocupación en México) (Figura 4).

A continuación, el segundo folio revestido del más grande valor documental para el presente estudio, contiene precisamente el nombramiento que otorga el Emperador Maximiliano con su firma autógrafa, al Profesor Don Eugenio Bergeyre, como Veterinario Principal del Cuerpo de Sanidad Militar del Ejército Imperial Franco-Mexicano firmado en el Castillo Imperial de Chapultepec, el 7 de septiembre de 1866 (Figura 5). ${ }^{d}$

d Ambos documentos se hallan en la Dirección General de Archivo e Historia de la Secretaría de la Defensa Nacional. Caja No. 63.D/III.6/1080. Año de 1935. 


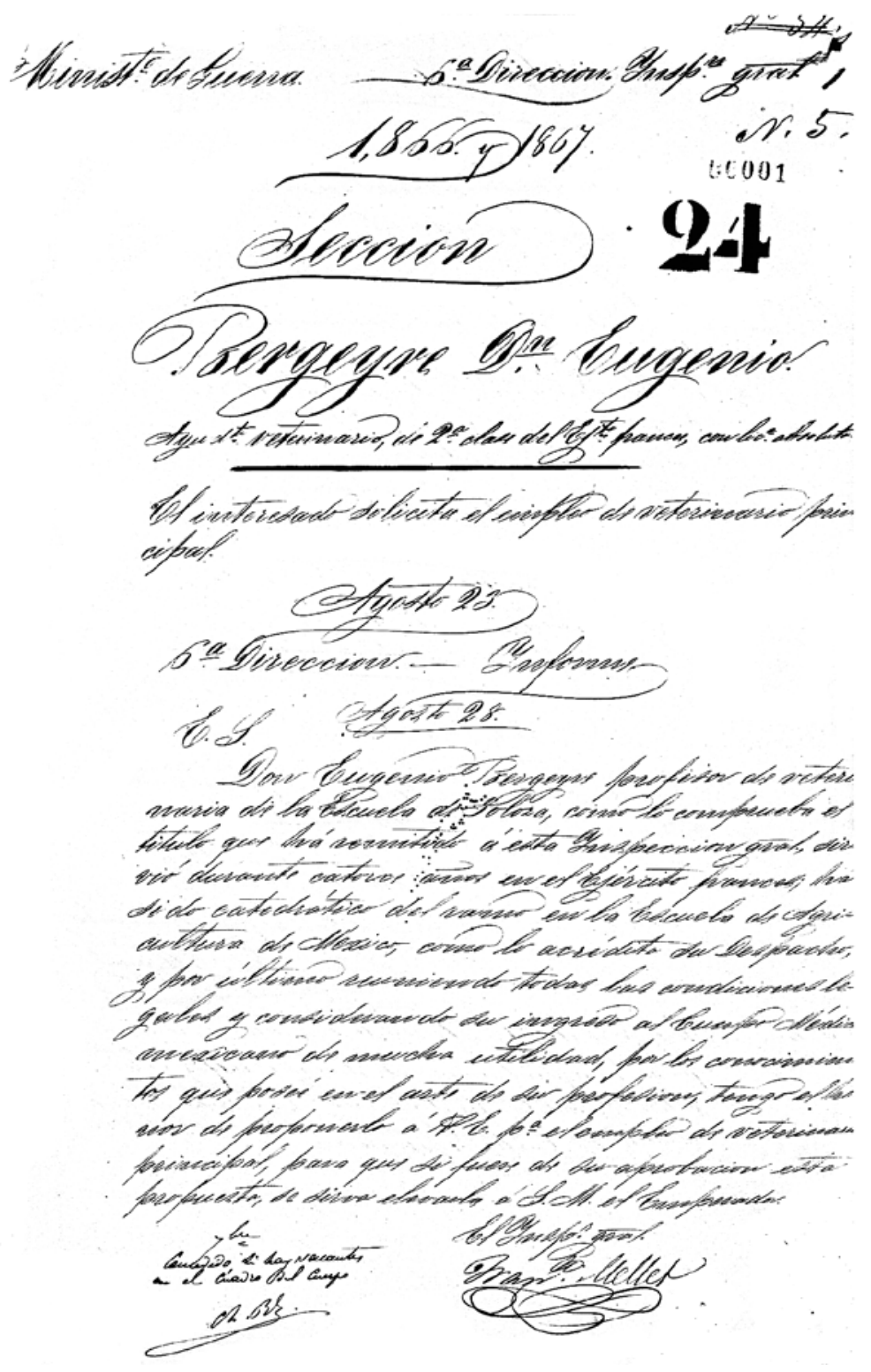

Figura 4.

\section{Transcripción:}

Ministerio de Guerra. $6^{a}$ Dirección. Inspección General
1,866 y 1,867
(No. 5.0001)

Sección 24

BERGEYRE, Dr. EUGENIO

Ayudante Veterinario de $2^{a}$ Clase del Ejército Francés

El interesado solicita el empleo de Veterinario Principal

Agosto 23

6. Dirección ----- Informe

Agosto 28

E.S.

Don Eugenio Bergeyre Profesor de Veterinaria de la Escuela de Tolosa, como lo comprueba el título que ha remitido a esta Inspección General, sirvió durante catorce años en el Ejército Francés, ${ }^{e}$ ha sido catedrático del ramo en la Escuela de Agricultura de México, como lo acredita su Despacho y por último reuniendo las condiciones legales y considerando su ingreso al Cuerpo Médico mexicano de mucha utilidad, por los conocimientos que posee en el arte de su profesión, tengo el honor de proponerle a S. E., el empleo de veterinario principal, para que si fuere de su aprobación esta propuesta, se sirva elevarla a S. M. el Emperador.

El Inspector General François Mellet

e Suponemos que se trata de un error de transcripción, debería leerse: "Ejército Mexicano" 


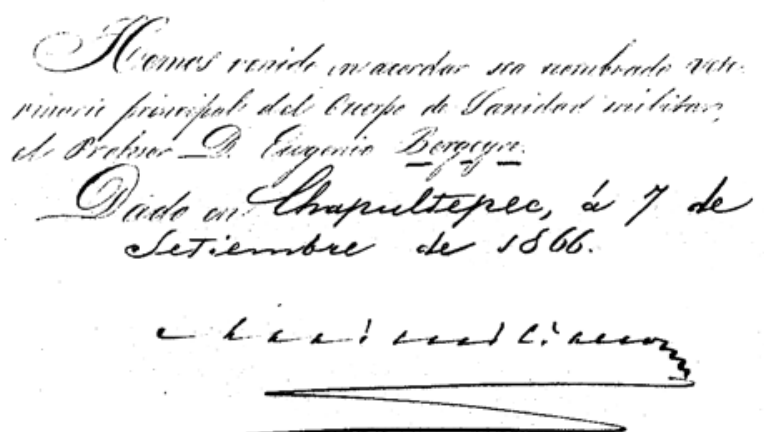

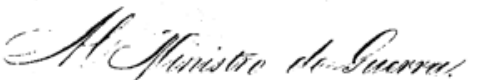

\section{Transcripción:}

Hemos tenido en acordar sea nombrado veterinario principal del Cuerpo de Sanidad Militar al Profesor D. Eugenio Bergeyre

Dado en Chapultepec, à 7 de Septiembre de 1866

Firma autógrafa del Emperador Maximiliano 


\section{Miembro de la Comisión Científica Francesa en México}

Carlos Luis Napoleón Bonaparte III, sobrino de Napoleón Bonaparte, en un intento de emular al gran corso cuando envió a Egipto junto con el ejército francés, a una comisión cientifica y cultural con el objeto de estudiar las riquezas del país de los faraones, hace lo propio en México. Francia necesitaba que su invasión militar a México estuviera avalada por intelectuales de prestigio que hicieran más aceptables los hechos y, a la vez, éstos podrían recabar información acerca del país, de sus riquezas naturales, mineras, agrícolas, ganaderas, arqueológicas y artísticas, entre otras. Se trató, en efecto, de una invasión militar imperial con características ilustradas. Ya antes de la ocupación de México, el emperador francés había creado por iniciativa de su ministro de la Instrucción Pública, Víctor Duruy, la "Comisión Científica, Literaria y Artística de México" cuyo reglamento fue editado en 1864, fundada en Paris por decreto de Napoleón III, del 27 de febrero de 1864 con el nombre de "Expedition Scientifique du Mexique".

Las credenciales y el prestigio como académico y científico que le investían, hicieron que el Dr. Eugenio Bergeyref, fuera invitado a formar parte de la mencionada comisión integrada por 150 especialistas de las más diversas ramas de la ciencia, arte y literatura (Figuras 6 y 7 ). Al inaugurarse los trabajos en ciudad de México, el 19 de abril de 1864 a las 12 del día, en el gran salón de actos de la Escuela de Minería, el General François Achille Bazaine, dirigiéndose a la Sexta Sección (de Medicina, Cirugía e Higiene) mencionó:

Médicos, enseñad las precauciones higiénicas con que se pueda preservar la salud del indígena, lo mismo que la del europeo, contra los peligros y vicisitudes que la amenazan en climas excepcionales, y señalad los medios con que se pueda combatir y vencer los males que la afligen, uniendo vuestros esfuerzos con los profesores del arte farmacéutico, descubrid las propiedades benéficas de los específicos que ofrecen los simples del Anáhuac y que conocidos de los indios todavía se hallan ignorados de los europeos. ${ }^{16}$

\section{Miembro fundador de la Academia Nacional de Medicina de México}

Una consecuencia de las primeras acciones de la Comisión Científica, Literaria y Artistica francesa en México fue la creación de la Academia Nacional de Medicina (ANM). ${ }^{17}$ El grupo estuvo integrado por veinticuatro miembros, de los cuales diecinueve eran médicos, entre los que se encontraban Manuel Carmona y Valle, José Ignacio Durán, León Condet, Luis Hidalgo y Carpio y Luis Muñoz, entre otros. Los farmacéuticos fueron algunos franceses: Jacobo Benoti, Alfonso I. Mercher y el mexicano Victoriano Montes de Oca y dos médicos veterinarios franceses: Eugenio Bergèyre y Constant Leguistin.

f En el listado que se halla en el expediente de Eugenio Bergeyre en el Archivo Histórico de la Secretaria de la Defensa Nacional, se menciona el nombramiento como Socio de la Comisión Científica de México (documento que se encuentra extraviado). 


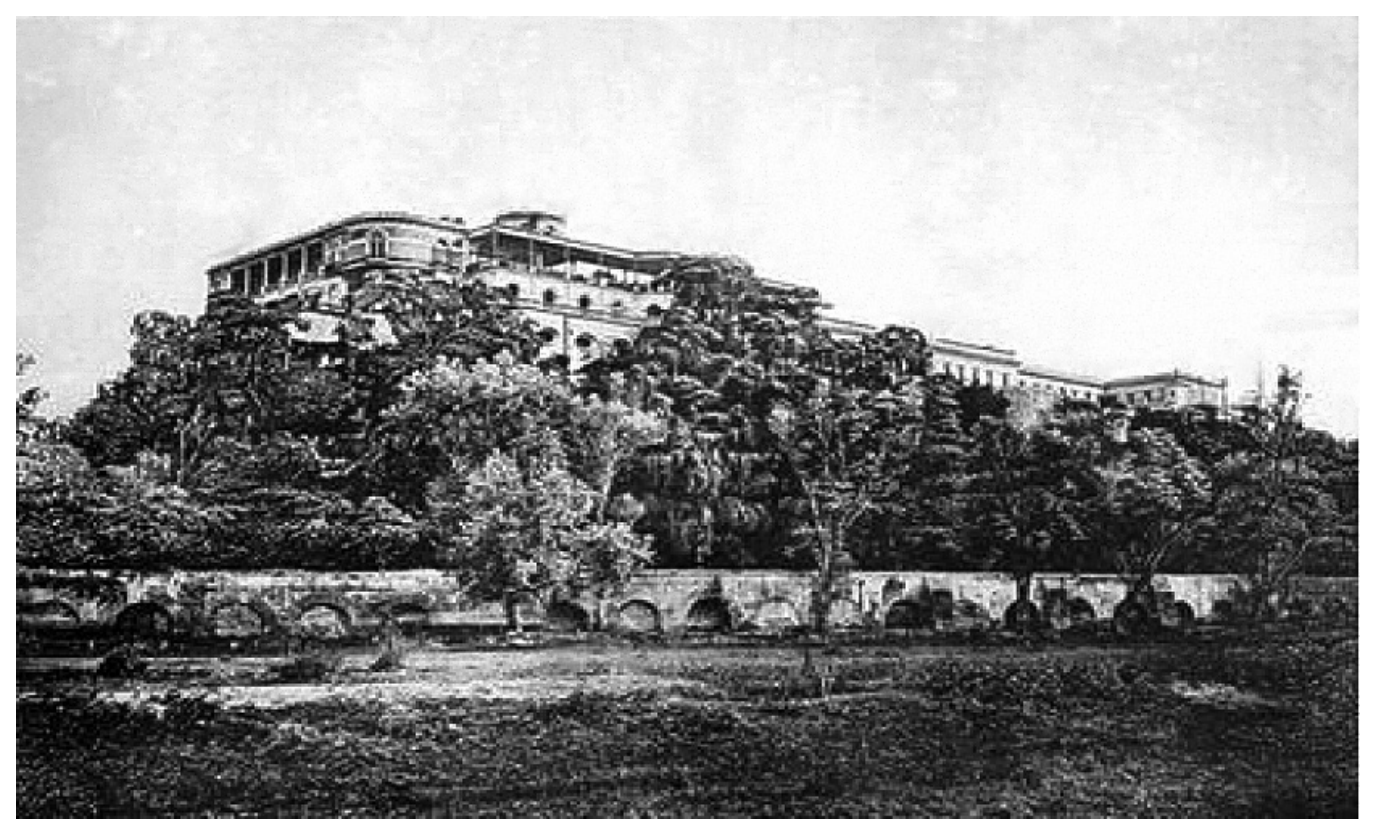

Figura 6. Castillo Imperial de Chapultepec. Ciudad de México. Circa 1866.
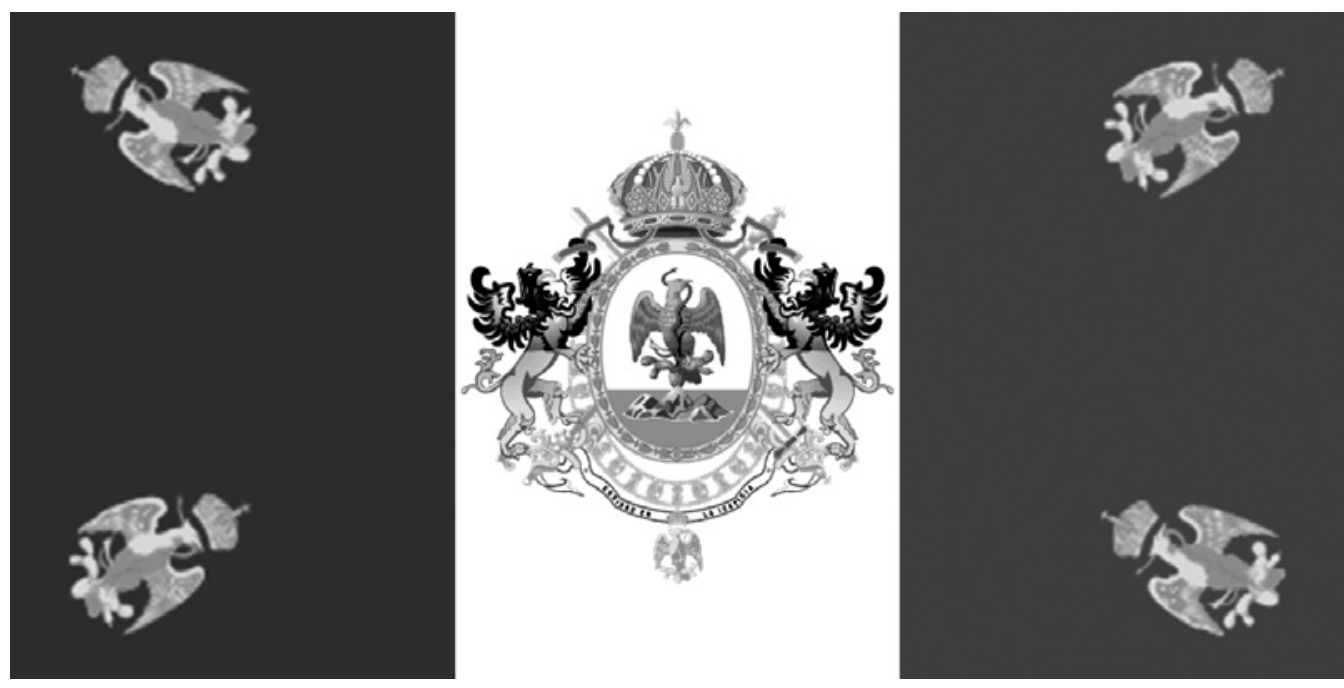

Figura 7. Bandera del II Imperio Mexicano de Maximiliano de Habsburgo 
Sus áreas de estudio fueron Patología, Higiene, Medicina Legal, Estadística Médica, Medicina Veterinaria, Materia Médica, Farmacología, Fisiología y Antropología. La primera mesa directiva estuvo conformada de la manera siguiente:

Presidente: Dr. Carlos Alberto Ehrmann, (médico militar).

Primer vicepresidente: Dr. Julio Clement

Segundo vicepresidente: Dr. Miguel F. Jiménez

Primer secretario: Dr. Carlos Agustín Schultze

Segundo secretario: Dr. Agustín Andrade

Tesorero: Dr. Rafael Lucio. ${ }^{18}$

Inmediatamente después del inicio de los trabajos, el 15 de febrero del 1864, se inició la publicación de la revista científica Gaceta Médica de México, cuyos artículos continúan publicándose hasta nuestros días, como órgano oficial de la Academia Nacional de Medicina de México. ${ }^{19,} 20$ Solamente hemos sido capaces de encontrar una publicación del Dr. Bergèyre en dicha revista, sobre el "Tifo Carbonoso" 21 y del Dr. Leguistin, igualmente con sólo un artículo sobre "Cowpox".22

Bergeyre, tiene fecha de ingreso a la Academia Nacional de Medicina de México del 30 de abril de 1864, con el sitial Número 4, cuando presentó su trabajo de ingreso. En esos días, el Dr. Bergeyre estaba domiciliado como "albéitar" en la calle de Alconedo No. 5 de la ciudad de México, según consta en el Directorio del Imperio. ${ }^{23}$

Otro dato curioso es el que el Dr. Bergèyre fungió como sinodal durante el examen profesional del Dr. José de la Luz Gomez, distinguido veterinario y científico mexicano quien se tituló como Profesor Veterinario en 1862 en la Escuela Nacional de Agricultura y Vetertinaria de San Jacinto.

\section{Bergeyre, el pater familias}

En 1880, después de una permanencia de 27 años en suelo mexicano, se apresta a retornar a su querida y añorada Francia. Antes de su partida contrae matrimonio en febrero del año arriba mencionado con doña Felipa Soto, dama mexicana con quien ha procreado cuatro hijos, Eugène Louis, nacido en 1869; Pascal Victor, nacido en 1872; Amédée Charles, quien ve la primera luz en 1873, y Marie Françoise, nacida en 1876 (Figura 8).

En un último correo dirigido a su hermano Próspere, antes de embarcarse en Veracruz el 15 de marzo de 1880, le informa de sus problemas económicos en México:

Je n'emporterais du Mexique qu'une chose, la pauvreté, mais une pauvreté honorable et sans tache. Dieu est témoin que je lutte, depuis trois ans, contre tous les déboirs qui attendent les Européens dans le Mexique. Ces trois ans de vaines espérances, de soucies sans nombre, de dépenses sans fins, m’ont rendu la vie annone, ont réduit mon avoir à rien...

(Yo no me llevaré de México más que una cosa, la pobreza, pero una pobreza honorable y sin mancha. Dios es testigo que yo lucho desde hace tres años, contra todos los deberes que esperan a los europeos en México. Estos tres años de vanas esperanzas, de inquietudes sin nombre, de gastos sin fin, me han rendido la vida vacía, me han reducido a tener nada...) 


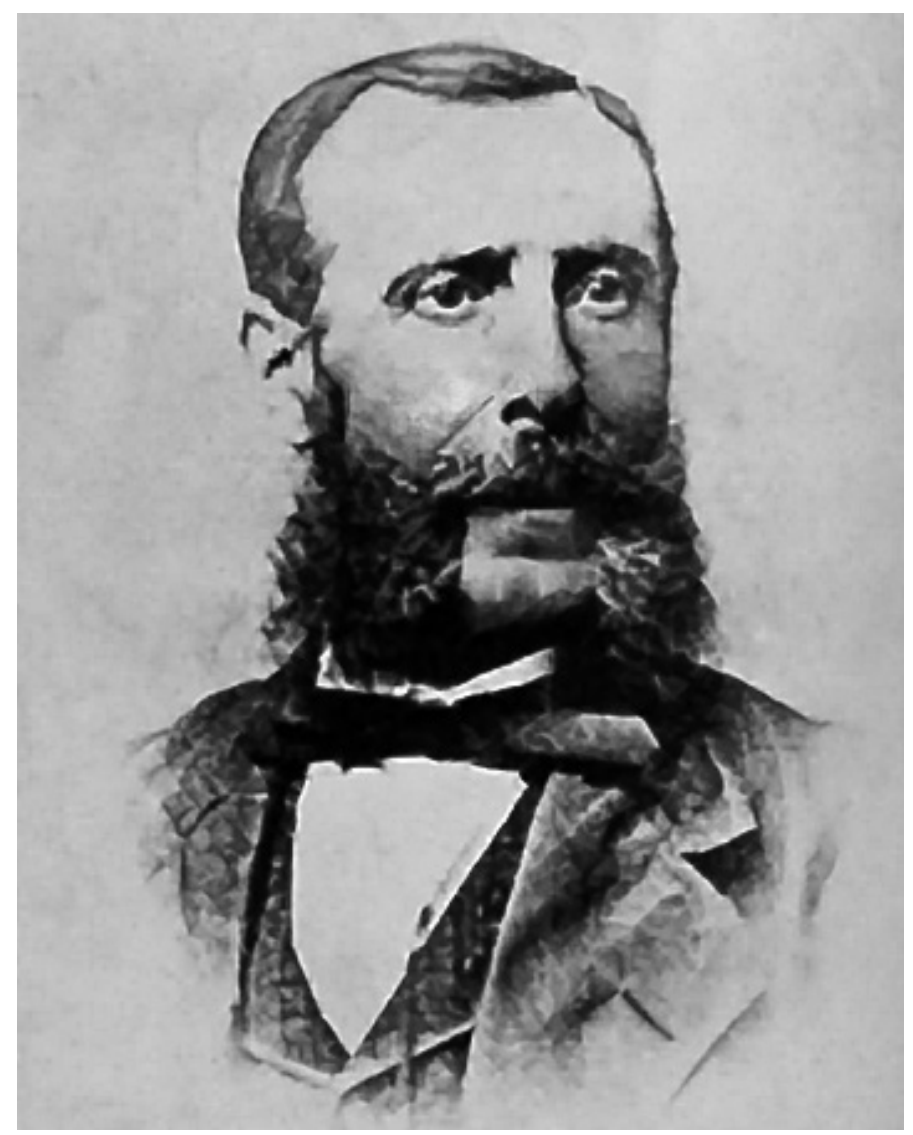

Figura 8. Dr. Eugène Bergeyre. Ciudad de México. Circa 1866

El reencuentro con su natal país vasco fue muy breve, ya que Eugène y su hermano Pascal murieron en el otoño de 1880, así como su esposa Felipa, en 1882. También Víctor perdió la vida en 1885, sin descendencia. Sus hijos Eugenio y Víctor retornaron a México en 1886 y 1888, respectivamente. Contrariamente a su padre, Eugenio y Víctor echarán raíces, serán prósperos negociantes y formarán sus respectivas familias, durante la época de oro del Porfiriato (Figuras 9 y 10).

Una veintena de sus descendientes habitan ahora en ciudad de México y en diversas partes del país, y la mayoría de ellos portan el apellido Bergeyre.

\section{Agradecimientos}

Deseo reconocer y agradecer en forma muy especial al joven Ingeniero Eugenio Felipe Bergeyre y Flores, descendiente directo del Dr. Eugène Bergeyre, quien nació, habita y labora actualmente en la ciudad de México. Eugenio fue un factor fundamental y clave en el proceso de investigación del pasado de su tatarabuelo. Con su apoyo y gracias a la valiosa documentación que me proveyó, fui capaz de reconstituir el pasado y la vida de primer veterinario civil y militar, que ejerció la medicina animal en nuestro país. Estos meses de intenso trabajo se convirtieron en el sustrato fértil, para que naciera una agradable y sincera amistad entre nosotros. 


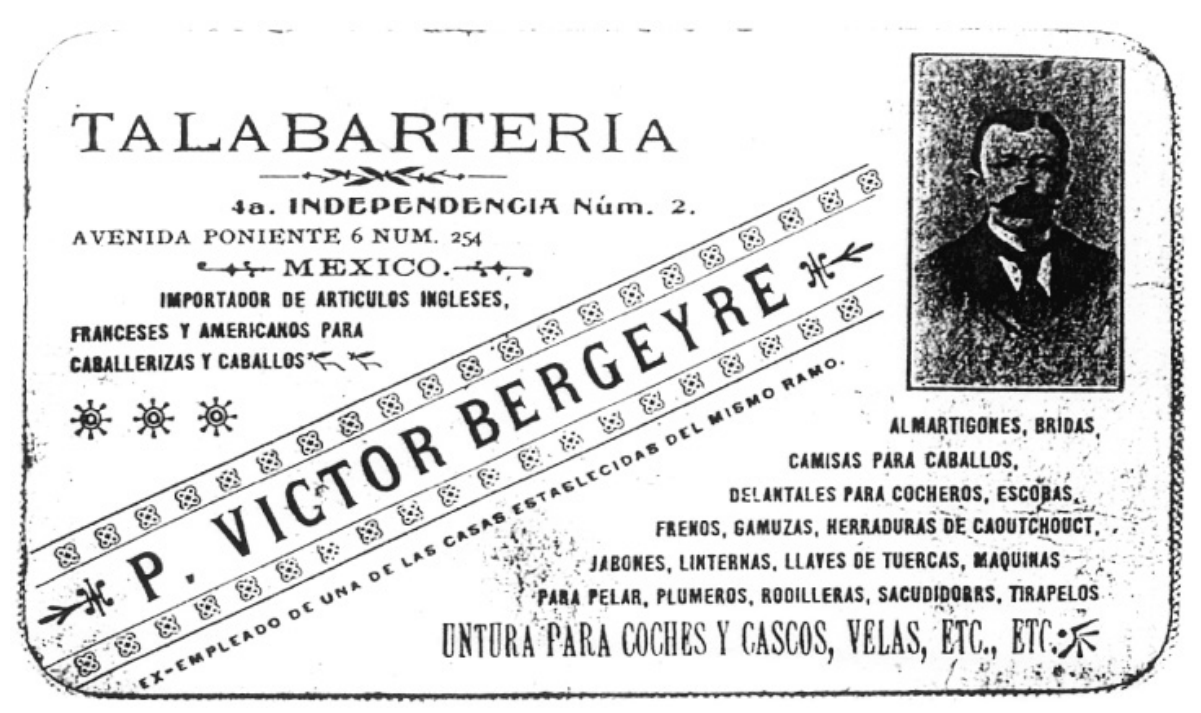

Figura 9. Negocio de Talabartería e importación de artículos para caballos, caballerizas y coches de tiro animal, propiedad de Pascal Víctor Bergeyre, hijo del Dr. Eugène Bergeyre, ubicado en la Calle de Independencia No. 4, Ciudad de México, hacia 1890.

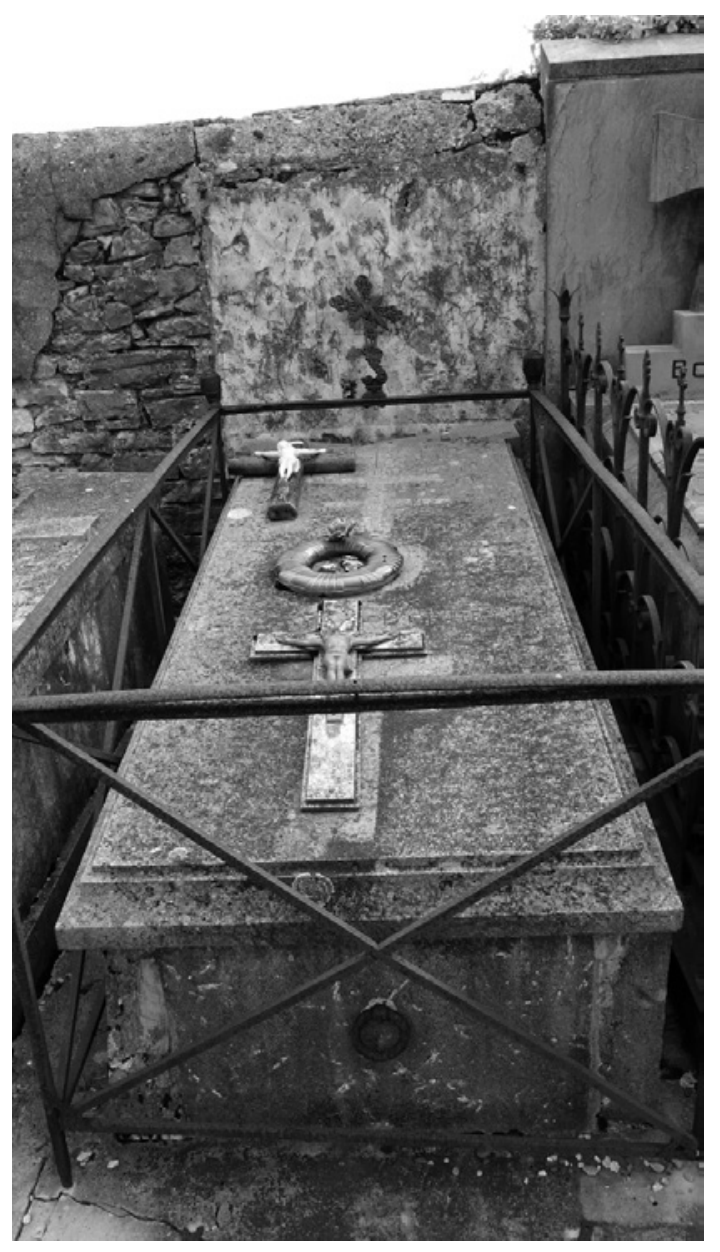

Figura 10. Tumba del Dr. Eugène Bergeyre y de su esposa doña Felipa Soto Cementerio de Saint-Leon. Bayona, Francia. 
Deseo también agradecer al Sr. Daniel Wiard de Langlade, Nîmes, genealogista vasco-francés descendiente también de nuestro biografiado, al Sr. Xavier Cabantous, genealogista de Bayona, a la Sra. Elena Lucaci-Martínez de Paris, igualmente descendiente del Dr. Bergeyre, al Sr. Meriadec de Goüyon de Matignon de Paris, pues gracias a él y a la Blibliotèque Nationale de France en Tolbiac, pude llegar a la primera evidencia escrita de nuestro personaje y a la Dra. Carmen Vázquez Mantecón del Instituto de Investigaciones Históricas de la UNAM, quien me reveló la primera señal del camino para documentar la saga de la "Aventura Mexicana de Eugène Bergeyre".

Finalmente vayan mis agradecimientos a los Médicos Veterinarios, Teniente Coronel Héctor Segura Medina, Teniente Coronel Armando García López y al Coronel Josué Navas Cervantes, Jefe del Servicio de Veterinaria y Remonta del Ejército Mexicano, quienes amablemente me abrieron las puertas del Archivo Histórico de la Secretaría de la Defensa Nacional. 


\section{References}

1. Bergeyre EV, Wiart D. Standard Pedigree Tree. Francia: Cercle Généalogique du Languedoc; 1989-2000.

2. Wiart $D$, Cabantous X. L'Aventure Mexicaine d'Eugène Bergeyre. Cercle Généalogique du Languedoc. 2007; 4a trimestre (117):30.

3. Archivo Histórico de la Secretaría de la Defensa Nacional. México, D.F.: Departamento de Dirección General del Archivo Militar. Secretaría de Guerra y Marina. Caja Número 63. D/III.6/1080. Expediente 00015. Año de 1935.

4. Documento proporcionado por el Sr. Daniel Wiart; 2009.

5. Bergeyre y Flores E. Archivo de la familia Bergeyre en México, D.F. y en Bayona, Francia.

6. Wiart y Cabantous. La Aventure Mexicaine... Carta citada.

7. El Siglo Diez y Nueve. México, lunes $1^{\circ}$ de agosto de 1853. Tomo Séptimo. Número 1682. Hemeroteca nacional. Fondo reservado. México: UNAM.

8. García Delgado G. Primer anuncio de servicios veterinarios ofertados en un periódico en México. Memorias de la Primera Jornada de Historia de la Medicina Veterinaria y Zootecnia. México: Facultad de Medicina Veterinaria y Zootecnia. Universidad Nacional Autónoma de México, 24 y 25 agosto 2000. p. 54-5.

9. Madame Calderón de la Barca. La Vida en México, durante una residencia de dos años en ese País. $1^{\text {a }}$ ed. México: Porrúa, Colección Sepan Cuantos; 1967. p. 30-1.

10. Barreiro A. Reseña histórica de la enseñanza agrícola y veterinaria en México. México: Tipografía El Libro del Comercio; 1906. p. 11.

11. Ramírez Valenzuela M. El establecimiento de la primera escuela de agricultura y veterinaria en México. Sus antecedentes y primera etapa de vida. Veterinaria México. 1978;12.

12. Flores y Troncoso F de A. Historia de la medicina en México, desde la Época de los indios hasta el Presente [tesis de la Escuela Nacional de Medicina]; 1888. México: Instituto Mexicano del Seguro Social. Segunda Edición Facsimilar; 1982. Tomo III. p. 247.

13. Uribe MBI, Cervantes Sánchez JM, Román AM. Capítulo II. Una nueva profesión se gesta en México. En: Cervantes SJM, ed. Una mirada a la historia de la medicina veterinaria a través de la vida y obra del Dr. José de la Luz Gómez. J. M. MP. 12. México: Facultad de Medicina Veterinaria y Zootecnia. Universidad Nacional Autónoma de México; 2010. p. 12.

14. Uribe MBI. La profesionalización de la medicina veterinaria a través de los trabajos y los días de José de la Luz Gómez. Tesis de licenciatura. México: Facultad de Filosofía y Letras, UNAM; 2008. p. 44.

15. Kolonitz P. Un viaje a México en 1864. $1^{\text {a }}$ ed. México: Colección SepSetentas; 1976. p. 74.

16. Muestra sobre "La influencia de francia en la medicina Mexicana". Excelsior, 16 de mayo de 1981.

17. Fernández del Castillo F. Historia de la Academia Nacional de Medicina en México. México: Ediciones Fournier; 1956.

18. Academia Nacional de Medicina. Presidentes 1864-2006. México: ANM; 2006. p. 7.

19. Sanfilippo J. Comunicación personal. México, 15 de febrero, 2010 
20. Diccionario Porrúa de Historia, Biografías y Geografía, $6^{a}$ ed. México: Porrúa; 199

21. Bergèyre E. El tifo carbonoso. Gaceta Médica de México. Tomo I. 1864-1865.

22. Leguistin A. El cowpox. Gaceta Médica de México. Tomo I. 1864-1865.

23. Directorio de Comercio del Imperio Mexicano; 1867. p. 248. 